\title{
Propagating Trusted Execution through Mutual Attestation
}

\author{
Furkan Turan \\ COSIC, KU Leuven (Belgium) \\ furkan.turan@esat.kuleuven.be
}

\author{
Ingrid Verbauwhede \\ COSIC, KU Leuven (Belgium) \\ ingrid.verbauwhede@esat.kuleuven.be
}

\begin{abstract}
Attestation is used in a trusted computing context to verify the expected good behaviour. It defines a prover and verifier relationship. It requires the presence of an authority if any two devices want to question each other for a sensitive collaborative work. In contrast, the mutual attestation defined in this paper allows the same rank and type devices to directly evaluate each other, and manage their own cooperation. In addition, it provides the attestations with response actions so that a device with an untrusted state can be recovered with the help of others on the network. These features are supported with a Root-of-Trust (RoT) module capable of enforcing actions even on exploited devices. A proof-ofconcept FPGA implementation of the proposed RoT module is demonstrated on Zynq SoCs, as an add-on extension to a RISC-V processor for establishing trust between applications of connected devices. Besides, it assists to explore the implementation decisions, abnormal execution situations, resource utilisation, and performance overheads.
\end{abstract}

CCS Concepts - Security and privacy $\rightarrow$ Embedded systems security.

Keywords Trusted Computing, Attestation, Security

\section{ACM Reference Format:}

Furkan Turan and Ingrid Verbauwhede. 2019. Propagating Trusted Execution through Mutual Attestation. In 4th Workshop on System Software for Trusted Execution (SysTEX '19), October 27, 2019, Huntsville,, ON, Canada. ACM, New York, NY, USA, 6 pages. https: //doi.org/10.1145/3342559.3365334

\section{Introduction}

Trusted computing relies on attestation to make a device prove its state, code or data to a remote party. For example, Intel's SGX [2] enables applications to start a process in an

Permission to make digital or hard copies of all or part of this work for personal or classroom use is granted without fee provided that copies are not made or distributed for profit or commercial advantage and that copies bear this notice and the full citation on the first page. Copyrights for components of this work owned by others than the author(s) must be honored. Abstracting with credit is permitted. To copy otherwise, or republish, to post on servers or to redistribute to lists, requires prior specific permission and/or a fee. Request permissions from permissions@acm.org. SysTEX '19, October 27, 2019, Huntsville,, ON, Canada

( 2019 Copyright held by the owner/author(s). Publication rights licensed to ACM.

ACM ISBN 978-1-4503-6888-9/19/10 ..\$15.00

https://doi.org/10.1145/3342559.3365334 enclave, and proves the integrity of that enclave to a remote party with attestation. Another example is Sancus [6] which targets embedded devices. In its design, a software module attests its state to its provider with a special instruction. Both SGX's and Sancus's attestation mechanisms force the involved parties to act honest, hence they obstruct malicious use. However, they do not define how to handle attestation failures. A failure often indicates a dishonest or malevolent use of a device. Unfortunately, who may own such a device cannot be controlled, e.g. Sancus enabled devices have been proposed for vehicles and smart meters [4]. In contrast to these examples, this work focuses on evaluating attestations, responding to them and handling their failures. Thus, the main contribution of this work is the attestation evaluation and corresponding responses. The evaluations yield decisions which include, besides binary decisions if a device is trusted, the suspicions if it malfunctions or if it is under an attack. The responses aim at actions to recover from an untrusted state when it is encountered.

Existing attestation mechanisms are defined for a hierarchy, where an inferior proves its state to an upper. In this relationship, the upper has the ability to validate the attested state with cryptographic verification. Though the hierarchy is useful in many applications, it prevents the use of attestation directly between two same ranked parties, who wish to know each other's state e.g. to share secrets or to collaborate on a sensitive job. It also makes the mechanism dependent on the availability of the TTP.

Our work argues that a more recent version of a code is more trusted, because it addresses known vulnerabilities. Therefore, our mutual attestation mechanism make devices evaluate each other based on the version of resources they have. Moreover, it enables the trusted party to force certain actions on the untrusted such as forced or postponed updates.

\section{Target Applications}

This work targets resource constrained device architectures that demand secure collaboration with each other.

Many-Core Architectures Connected small cores achieve big computation power through collaboration. For example, InvasIC [9] proposes a novel architecture with a dedicated programming paradigm. The architecture is empowered by connected many tiles of processors. These tiles are the resources for programs. The tiles are dynamically allocated 
to them at run time according to the demand. In the InvasIC context, a program invades the neighbouring tiles, when it requests more processing power. Then it claims the invaded resources and transfers a part of its job. When these resources are redundant, the program retreats, making them available for another program's invasion. As the computation is distributed dynamically, it is necessary to know if the invaded nodes are trusted especially when sharing critical jobs. A code executed previously on an invaded tile may have altered its system state maliciously. Likewise, the programs may receive security updates at run time, which may pose vulnerabilities if not available yet at the invaded tiles.

Mesh Network Applications Smart meters allow the remote metering of energy consumption for a better balanced grid and lower energy production cost. The mesh network topology is a strong candidate to connect them [3]. However, the security of meters and their network is a concern. Preventing their improper use is required for increasing the network robustness and make them reliable to each other. Ensuring their availability is as important as protecting them from attacks according to their security analysis [5]. Sancus [4] is an ideal platform to build a high-assurance meter. Relying on the attestations, it aims at detecting the potential malice of the meter holder e.g. for reducing bills or damaging the infrastructure. Our proposed propagating trust mechanism improves these attestations by fixing the malicious modifications, and making the meters run the most up-to-date software.

\section{Assumptions \& Attacker Model}

We assume that individual devices (i.e. cores in a manycore setting or meters in the mesh network) are untrusted and might exhibit arbitrary behaviour. Even though wellbehaving, they can be compromised, loosing the complete control of their functionality and/or cryptographic material. The devices' communication medium is also assumed untrusted. We make no assumptions about the number of malicious devices in the network, their neighbourhood, or the connectivity between them. We assume the existence of a trusted source e.g. an administrator who is inherently trusted and can update devices' knowledge of trusted content providers.

Different kinds of adversarial behaviours are covered. An adversary can inject malicious binaries, code, or configuration into the devices. It can yield the complete control of a device, at a minimum enforcing it to deny service. It can eavesdrop on the communication of devices in the system. Finally, it can try to convince other devices on the network and send arbitrary attestation messages.

\section{Measurements}

In the trusted computing context, a measurement is used to evaluate the state of a chosen binary or memory content.

\begin{tabular}{|c|c|c|c|}
\hline Metadata & Signature & Content & Signature \\
\hline
\end{tabular}

Figure 1. The files contain metadata appended with the object provider's signature. The metadata is a part of the object's content, and the content is signed again by the provider.

State-of-the-art considers object hashes as their measurement, which is only applicable when acceptable hash values are known beforehand. This complicates making devices evaluate each other. Provisioning devices with the exact same copy of the objects is not possible in real life. Therefore, the devices should be able to verify each other's measurements even when the objects they hold differ. For example, when a device receives an update, other devices that have not received it yet, don't know how to validate the received measurement of the updated. Hence, we opt to describe the objects' specifics together with its measurement.

Our measurement mechanism provides the objects with metadata and cryptographic signatures. As shown in Figure 1 , an object consists of a content and its signature, the content involves a metadata that has its individual signature. Both signatures are prepared by the object's provider. The outer signature is for the local verification of the object handled by the RoT hardware. If the verification is successful, the metadata is taken as the measurement subject to attestations. The metadata signature is used by the attestations to prove the measurements' validity. Further sections of this paper uses the verb measuring for signature verification, and the noun measurement for the metadata of verified objects.

The attestations of measurements arrive to a decision by comparing them. Hence, the measurements (i.e. the metadata) should contain enough information for a thorough comparison to decide which one is trusted, or more trusted. In this respect, we propose to start with an objects' version. We assume that a recent version of code is more trusted, because it has addressed known vulnerabilities and has issued security updates. The version information is extended with the identifiers of the object and its provider. The latter is necessary to know whose signature will be verified.

Software updates do not always target security issues, but sometimes extend device features. Besides, an assessment of what features that an old software version cannot be trusted is possible, because the vulnerabilities of it are often known. Hence, it is possible to decide to severity of updates, and postponing them for minimising their overhead on performance. In addition, the evaluation is extended with software dependencies. For example, an application may depend on a set of OS features. Hence, it trusts the neighbours if they have at minimum a certain version of the OS which implements these features without known vulnerabilities. In such a case, the neighbour may not have the most recent version of the OS, but it can still be trusted for the specific application. Hence, the metadata is extend to include the dependencies with their object and provider identifiers, and their version. 
Propagating Trusted Execution through

Mutual Attestation

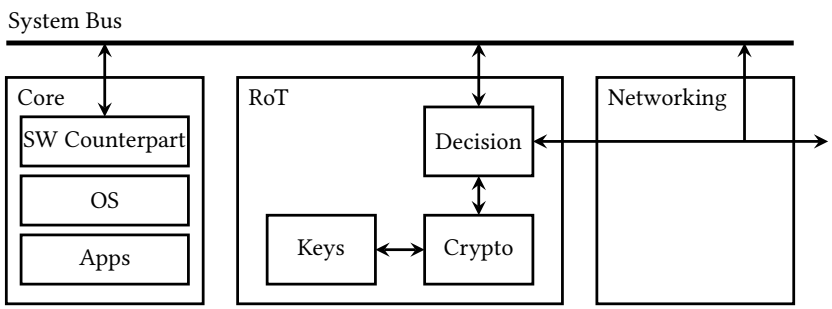

Figure 2. The RoT hardware uses crypto module to verify the attestation messages, networking for communicating them, and decision unit for attestation responses and enforcements.

Various other information can be preferred over version for trust evaluation. For instance, dudect [7] tool provides statistical timing leakage analysis of applications. The comparison of binaries can be held according to their score, and the one with minimum leakage could be preferred. Similarly, Information Flow Control (IFC) analysis, e.g. made with $\mathcal{F O A N A}$ [8], can be used to compare the leakage score of binaries.

\section{Mutual Attestations and Responses}

We propose to make devices measure their resources, and exchange them with their neighbours. This exchange is the foundation of mutual attestations. It makes a device that sent its measurements to a neighbour receive measurements from the neighbour as reply. An evaluation follows the attestation and yields a decision i.e. a response to the completed attestation. A response hopefully lets the devices trust each other and collaborate, but may also reveal that one has untrusted objects compared to the other. In such cases, the untrusted one receives either an immediate or postponed update. To perform these attestations and decision-making, we propose a Root-of-Trust (RoT) module as shown in Figure 2. This section describes the attestation capabilities of the module, the next section will shed light on its architectural details.

The proposed RoT module employs secure boot in the sense that the bootloader is subject to attestations and updates. The module halts the execution of the FSBL at power on, measures it and attests to neighbours. That is repeated for the next boot stages. As a result, the devices boot up to the most up-to-date execution environment possible. Besides, it creates a list with the measurements of bootloader of various boot phases. This list is referred as trust chain by the Trusted Computing Group in the context of TPM. We propose to use this chain at run-time attestations to define the execution environment. That strengthens the attestations between applications of neighbour devices with the search for the most trusted execution environment.

This bootloader approach offers advantages over the typical secure boot procedures that rely on Read-Only Memory (ROM). It allows updatable code rather than fixed.
SysTEX '19, October 27, 2019, Huntsville, ON, Canada

Besides boot-time, the attestations are executed at run time when applications question the state of neighbour devices or answers the neighbours' requests. For example, a message sender may demand a measurement from the neighbour if the integrity of the target application or device is critical. This requires the sender to attest itself or an object of interest to the neighbour first, and the neighbour replies in response. The RoT module handles the attestation of measurements and decision-making.

\subsection{Attestation Messages}

The basic message types are listed and described below.

Attestation Request. This is an attestation message with a purpose to receive a reply from a target neighbour with the attestation of its measurements. It involves the trust chain always. In addition, the message may involve a payload for the measurement of a particular object of interest for the run time attestations.

Attestation Reply. The RoT module, which receives a request, replies with an attestation message having the same structure as the request message.

With the reply, the RoT modules of neighbours learn each others measurements. Upon comparing them, the modules decide to a response action e.g. continue, update, reboot (i.e. elaborated in the next section). The decision may enforce an action on a device or a certain object of it, if found untrusted compared to the other. The action is performed only after receiving a proof for the measurement, which authorises the enforcement of the trusted over the other.

Attestation Response. This message contains the aforementioned decision and proof. It is enough if the proof is sent only by the party who claimed the trusted measurement, because the other party will be asked to perform the enforcement action. In addition, the response messages may be issued from both sides, enforcing an update action for different objects of each other.

\subsection{Responses \& Enforcements}

The three basic type of response actions are listed below. Potential abnormalities require additional actions, which will be described next.

Continue. If the compared measurements are equal or equally trusted, no special action has to be taken, the devices or applications can work together.

Update Enforcement. If a device owns a trusted version of an object (e.g. item from the chain, an application or data file) compared to other, the RoT receives it from the neighbour, and updates the object.

The RoT module preempts the execution for performing an update at run time (details will be given later). However, there might be situations that break the halted execution due to a 
dependency of running applications to the updated contents. For such situations, below two responses are considered.

Update Request. If the update of an object should be performed with the awareness of the OS, then the update is requested from it, only if the attestations find it trusted.

Reboot. If the update targets the OS or bootloader, then reboot is the only solution to perform the update.

In order to minimise the execution time overhead of updates, the decision should account the updates' severity. For example, non-critical updates can be postponed until a convenient time. We propose to consider the severity information with dependencies (described in Section 4).

\subsection{Abnormalities \& Special Responses}

An abnormal execution may be observed under an attack or at a fault, which makes the binary trust decision hard with the above given responses. These situations should be foreseen as well, and the corresponding reactions be defined carefully. The reaction to handle such conditions is given below, and its use is exemplified later.

Mark as Suspicious. A RoT module issues this marking as part of the response, if the expected attestation routine could not be followed, which should be caused by a severe problem, e.g. an attack in the worst case scenario. If a RoT module is issued with this marking, it conducts a new attestation to -if possible- another neighbour. It also informs the neighbour about the mark using the message of the new attestation. The module clears the marking when completing the new attestation with success, and doing the updates if needed. If an integrity issue or untrusted software is not found, a device failure can be suspected. Thus, the system is reinitialised with existing software that helps recover to a known initial state from failed. Within these new attestations, if the neighbours find that they have an equivalent trust chain to the marked device, they can suspect that a similar situation (attack or failure) may happen to them. In that case, they should mark themselves as suspicious, but with postponed reinitialisation if no software update is found, as their state is still intact.

The next paragraphs exemplifies two abnormalities and how to use the above given reaction for resolving them.

Attestation reply is not received The RoT modules exchange the request and reply messages directly, thus a reply must be received when delivering a request. Suspecting a network error, the RoT module should re-send the attestation request. A repeat of the problem indicates that the RoT module is stuck at preparing the reply as follows. When receiving an attestation request for an object, the module should reply with its measurement. If the object is not already available in the trust chain, the module should find and measure it. For required system filesystem operations, the module uses its software counterpart (described later). If the counterpart fails and does not respond, the reply cannot be prepared. To resolve this issue, the RoT module omits the awaited measurement, and replies the repeated request only with the trust chain, which includes the measurement of the software counterpart. This lets the requesting entity evaluate the received chain, and enforce updates if necessary. If no updates are available, both devices has an equivalent chain, so vulnerable to the fault or attack that triggered the problem. In that case, both devices are marked as suspicious, and follow the further steps this marking requires.

Attestation response with an invalid proof This situation is not expected, as the measurements are attested after verification. Also, the chain should be kept secure by the RoT module. Hence, if a proof is invalid, the receiver can first suspect of a network error corrupting the message. A repeat of the problem denotes the failure of the sender's RoT module. Example suspicions include corruption of its memory contents or software counterpart. Hence, it should be marked as suspicious for reinitialisation with trusted software.

\section{Architecture}

The proposed RoT hardware consists of three basic blocks as shown in Figure 2: (1) cryptography module for digital signatures, (2) key storage for its administrator's key, and (3) connection to the network interface module. The cryptography module implements a digital signature scheme for taking measurements, and for their verification. The networking module enables communication between the RoT modules of neighbour devices by forwarding the relevant packets directly to the modules when receiving them. In the further paragraphs of this section, architectural details are given for the key management to support the cryptography, and for the software counterpart.

Key Storage The RoT decides what is trusted with the stored administrator and content provider keys. The administrator is the inherently trusted entity, maintaining the devices and infrastructure. It owns a public and private key pair. The private key is its secret, used for signing a content. The devices know its public key for verifying its signatures. This key is the reason that devices trust the administrator, hence a device's knowledge of this key should be protected from manipulations. This can be achieved by burning the key into the devices at manufacture or installation time using a One Time Programmable (OTP) key storage, e.g. made of eFuses.

A key list is used for the public keys of trusted providers, i.e. third party application developers authorised by the administrator. This list consists of public data, so it does not need protection from being read. However, its entries are authenticated with administrator signatures. These keys can be kept in a dedicated memory of the RoT hardware, or in the device's non-volatile data storage.

Software Counterpart A software part is proposed to support the RoT hardware with filesystem operations such as 
Propagating Trusted Execution through

Mutual Attestation

Ethernet Bus

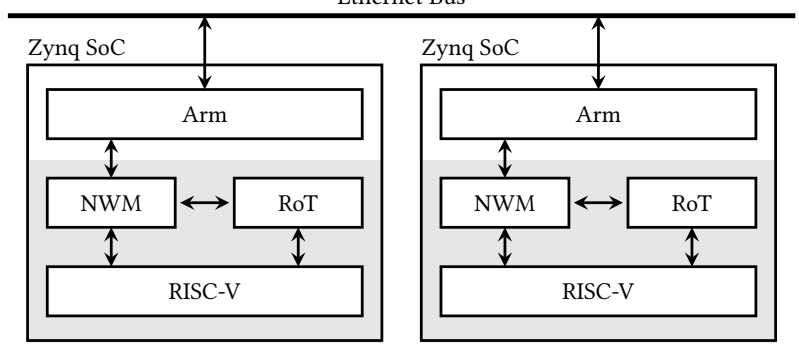

Figure 3. An FPGA implementation of RoT attached to a RISC-V and Networking Module (NWM) targets the FPGA of the Zynq, while NWM's IP stack on its Arm cores.

finding and accessing files for measuring or updating them. It executes with the highest privileges for providing the RoT with access to all system resources, and be protected from lower level software. However, the measurements, related cryptography and their communication are still handled only by the RoT hardware. Besides, the RoT keeps the counterpart intact by the attestations of it. Also, it is made a part of the FSBL that allows to verify it at the system initialisation, and to receive its help for verifying the next stage bootloaders.

\section{Implementation}

We prototyped our design to evaluate the proposed RoT module and explore the design decisions. It also allowed us to discover the abnormalities, and improve the design further. Our proof-of-concept implementation targets RISC-V processors, which boot Linux OS and run applications. As shown in the Figure 3, multiple instances are connected over Ethernet allowing the applications to communicate. They are implemented on Zedboards, employing a Zynq SoC device that integrates Arm processors with an FPGA. The untethered RISCV implementation of lowRISC [10] is chosen as the target processor. Our RoT hardware includes a lightweight implementation of Ed25519 [11] for the cryptographic operations. In addition, a custom networking module employs a hardware-software codesign for delivering messages to neighbours over an IP stack implemented on the Arm cores.

The design does not modify the RISC-V core with new instructions or features, but extends its first stage bootload (FSBL) routine. In the modified routine, the RoT verifies the FSBL by directly accessing the BRAM storing it, and allows its execution later. The RoT drives the reset signal to the processor at system power on for halting its execution until the FSBL's verification and attestation is complete. The module works alone in this initial stage, as its software counterpart becomes available only with the verified FSBL. If the verification fails, the RoT searches the neighbours for a verified one. Later, the same checks are made for the next level bootloader and OS binaries that the FSBL will hand over the platform.

The RISC-V Supervisor Binary Interface (SBI) is used to allow the user-space applications to communicate directly
SysTEX '19, October 27, 2019, Huntsville,, ON, Canada

with the software counterpart deployed in the machine level. That eliminates dependencies at the OS. When applications execute the mcall instruction, the corresponding handler in the SBI receives the call, and directs it to the counterpart together with the received arguments. Applications use this call to request attestations from the software counterpart, and they get the result of the executed attestations with a return value from the call. In addition, a background service on the OS is provided for the cases when an attestation ends up with a decision to update the application that initiated it. Such updates cannot be handled by the application itself or by the software counterpart because the lack of OS's awareness may break the execution.

The Ed25519 elliptic curve algorithm is used by the RoT module for the signature verification. Preferred implementation [11] is tailored for the same target FPGAs, achieving a lightweight hardware without demanding significant performance overheads. An advantage of Ed25519 algorithm is the high-level security with only 256-bit key sizes, which minimises the secret key storage size.

The attestations target ELF files, on which new sections are introduced for the metadata and signatures as shown in Figure 1. Each metadata consists of a chain of 32-bit elements. The file is described with the first element, and its dependencies with the next. Each element consists of two 10-bit identifiers for the file and its vendor, and a 12-bit identifier for its version. The signature sections are 512-bit long which is the size of Ed25519 signatures.

The concepts are demonstrated by connecting multiple small ZedBoards, each models one tile or node on the network. The networking module (NWM) offers a simple interface for the communication of applications running on the RISC-V cores, and for the attestation message transfers. The NWM is supported by the Arm cores with lwIP [1] (lightweight IP stack) to transfer messages over the Zedboard's 10G Ethernet interface to an other ZedBoard.

\section{Evaluation}

The following paragraphs evaluate the implementation respectively for its resource utilisation and performance.

Resource Utilisation The target Zedboard is a low-cost development board with a Zynq 7020 i.e. a small SoC device. The FPGA of the device is used to instantiate the RISC-V core and our RoT module. Table 1 provides the details of the FPGA resources required. The RISC-V is the largest component. The RoT module has a comparable size to the FPU. It consists of a sophisticated state machine, and a module for Ed25519 digital signature scheme. The Ed25519 module has a compact implementation that benefits from the FPGA's DSP slices to store computation data in them, and execute fast. Our prototype uses its base implementation; however, it offers options against fault and side-channel attacks which come at the cost of extra resource utilisation or performance 
reduction. These options can be enabled for the defined attacker model. Lastly, the Ed25519 implementation uses the SHA512 for taking hash of the input data, but it can be replaced with a hardware-resource-friendly hash algorithm.

Performance An evaluation of the performance consists of two major aspects, which are the execution time of measurements (signature verification) and the communication bandwidth. The former depends on the cryptographic implementation and the overhead of data access, while the latter depends highly on the used networking interface. In this section, we share the measurements of these two for our implementation. However, we would like to note that they - especially the latter- depend on the target device, and will vary for different systems.

The maximum clock frequency is limited to $25 \mathrm{MHz}$, which is set by a critical path within the RISC-V core. This core is not our custom implementation, but generated by the Rocket core generator. In contrast, the individual synthesis of the cryptographic hardware achieves $82 \mathrm{MHz}$.

The signature verification speed of the used Ed25519 hardware is $10 \mathrm{KiB} / \mathrm{sec}$ at $25 \mathrm{MHz}$. The FSBL is $135 \mathrm{KiB}$ and it is verified at this speed takes 13.5 seconds. In contrast, the further measurements are slower, as they depend on the software counterpart for accessing the system memory or SD card, and involves the overhead of interfacing between the hardware and software. As a result, verifying a $10 \mathrm{KiB}$ file system memory region takes around 3 second, while a same size file from the SD Card requires 10 seconds. The example is given for this size, because the applications of the target resource constrained devices are often small in size. For example, musl is a preferred compiler for them as a lightweight alternative to glibc and reduces application sizes from $\mathrm{MiB}$ to tens of KiB. Better verification speed is achievable with a processor that supports a higher clock frequency.

The NWM is used for the communication of RISC-V applications and RoT modules. The attestation messages are short UDP packages, as a list of 32-bit metadata, or 512-bit signatures. Their transfer take place if the previous transfers are complete. That simplifies the RoT state machine, which

Table 1. FPGA Resource Utilisation Results for Zedboard

\begin{tabular}{lrrrr}
\hline Available & LUTs & REGs & DSPs & BRAM \\
& 53200 & 106400 & 220 & 140 \\
\hline SoC & 46186 & 21551 & 32 & 65 \\
- RISCV Core & 31219 & 15259 & 16 & 65 \\
$\llcorner$ FPU & 11256 & 3231 & 11 & 0 \\
Memory Controller & $\ldots$ & $\ldots$ & $\ldots$ & $\ldots$ \\
- UART, SD Card & $\ldots$ & $\ldots$ & $\ldots$ & $\ldots$ \\
NWM & 1748 & 1457 & 0 & 0 \\
RoT & 11770 & 3076 & 16 & 0 \\
$\llcorner$ ED25519 & 11638 & 2615 & 16 & 0 \\
$\llcorner$ SHA512 & 2153 & 1555 & 0 & 0 \\
\hline
\end{tabular}

is better for an error prone design. In contrast, bulk-data transfers are used for file updates. The file is first streamed to the IP stack. The stack minimises the transfer time by managing the corresponding buffers and packet sizes. The software counterpart can stream a $1 \mathrm{MiB}$ file in 7.030 seconds, while that file is transferred to neighbour in 0.262 second.

\section{Conclusion}

In this paper, we describe an attestation mechanism allowing devices to question whether they can trust each other. It eliminates the need to an authority for this evaluation. The mechanism is extended with response actions recovering the device state from untrusted. Besides, the proposed mechanisms are supported with a proof-of-concept RoT hardware module implementation for a RISC-V processor.

\section{Acknowledgments}

This work was supported in part by the German Research Foundation (DFG) as part of the Transregional Collaborative Research Centre "Invasive Computing" (SFB/TR 89), the KU Leuven Research Council through C16/15/058, and ERC Advanced Grant 695305.

\section{References}

[1] 2010. lwIP - A Lightweight TCP/IP stack. https://savannah.nongnu. org/projects/lwip/.

[2] Victor Costan and Srinivas Devadas. 2016. Intel SGX Explained. IACR Cryptology ePrint Archive 086 (2016).

[3] Sabine Erlinghagen, Bill Lichtensteiger, and Jochen Markard. 2015. Smart meter communication standards in Europe-a comparison. Renewable and Sustainable Energy Reviews 43 (2015), 1249-1262.

[4] Jan Tobias Mühlberg, Sara Cleemput, Mustafa A Mustafa, Jo Van Bulck, Bart Preneel, and Frank Piessens. 2016. An implementation of a high assurance smart meter using protected module architectures. In IFIP International Conference on Information Security Theory and Practice.

[5] Mustafa A Mustafa, Sara Cleemput, and Aysajan Abidin. 2016. A local electricity trading market: Security analysis. In PES Innovative Smart Grid Technologies Conference Europe (ISGT-Europe), IEEE.

[6] Job Noorman, Jo Van Bulck, Jan Tobias Mühlberg, Frank Piessens, Pieter Maene, Bart Preneel, Ingrid Verbauwhede, Johannes Götzfried, Tilo Müller, and Felix Freiling. 2017. Sancus 2.0: A low-cost security architecture for IoT Devices. ACM Transactions on Privacy and Security (TOPS) 20, 3 (2017).

[7] Oscar Reparaz, Josep Balasch, and Ingrid Verbauwhede. 2017. Dude, is my code constant time?. In Design, Automation \& Test in Europe Conference \& Exhibition (DATE), IEEE.

[8] Gregor Snelting, Dennis Giffhorn, Jürgen Graf, Christian Hammer, Martin Hecker, Martin Mohr, and Daniel Wasserrab. 2014. Checking probabilistic noninterference using JOANA. it-Information Technology 56, 6 (2014).

[9] Jürgen Teich, Jörg Henkel, Andreas Herkersdorf, Doris SchmittLandsiedel, Wolfgang Schröder-Preikschat, and Gregor Snelting. 2011. Invasive computing: An overview. In Multiprocessor System-on-Chip. Springer, 241-268.

[10] Furkan Turan. 2017. Working with Zedboard. https://www.lowrisc. org/docs/debug-v0.3/zedboard/, last checked on 2019-08-20.

[11] Furkan Turan and Ingrid Verbauwhede. 2019. Compact and Flexible FPGA Implementation of Ed25519 and X25519. ACM Trans. Embed. Comput. Syst. 18, 3, Article 24 (2019). 